\title{
Profitable Chaos in a Duopoly Market with Asymmetric Production Externality
}

\author{
Akio Matsumoto* and Yasuo NonakA**
}

\begin{abstract}
This study investigates an economic implication of chaotic fluctuations that are observed in a nonlinear economic dynamic model. To this end, it constructs a nonlinear discrete time Cournot duopoly model in which firms have $U$-shaped or inverted $U$-shaped reaction functions due to production externality and shows that chaotic output fluctuations can arise for strong nonlinearities. Two main results of this study are: (i) it is theoretically as well as numerically confirmed that one of the duopolists can benefit in the sense that the long-run average profit taken along a chaotic trajectory is higher than the profit taken at an equilibrium point while the other is disadvantaged if both duopolists are homogeneous; (ii) it is verified with numerical simulations that both duopolists can benefit from chaotic trajectories if they are heterogenous.
\end{abstract}

\section{Introduction}

In the last two decades, it has been demonstrated that a Cournot tatonnement process may be chaotic if reaction functions are non-monotonic ; see Rand [13], Dana and Montrucchio [4], and Witteloostuijn and Lier [14] (WL hereafter), to name a few. Recently at least the following three questions, all of which are strongly related to nonlinearities involved in the Cournot model, have admittedly received increasing attention: (i) What are sources of non-monotonic reaction functions? (ii) How to select an equilibrium when multiple equilibria exist? (iii) What is a economic implication of a chaotic market?

The first question raises the implied question as to what is the microeconomic foundation for nonlinear reaction functions, and two answers have already been given. One is by Puu $[11,12]$ who shows that linear production technologies and a hyperbolic market demand may result in unimodal reaction functions. The other is by Kopel [7] who confirms that under different assumptions of a linear market demand and nonlinear cost functions, reaction functions can be unimodal. The second question naturally arises because multiple equilibria are quite possible under non-monotonic reaction functions. Bischi and Kopel [2] deal with a problem of equilibrium selection and verify that the selection strongly depends on the choice of initial states of the dynamic process. The third question is partly answered for a one-dimensional dynamic process. Huang [6] makes clear that a chaotic cobweb market can be preferable to an equilibrium state. Matsumoto [8] points out the possibility that a

\footnotetext{
* Department of Economics, Chuo University 742-1, Higashi-Nakano, Hachiohji, Tokyo, 192-0393, JAPAN E-mail : akiom@tamacc.chuo-u.ac.jp

** Department of Economics, Kanto Gakuin University 1-50-1, Mutuura-Higashi, Kanazawa-Ku, Yokohama 236-8501, JAPAN E-mail : nonaka@kanto-gakuin.ac.jp
} 
pure exchange economy with two goods and two agents is better off along chaotic fluctuations than in the stationary state in the long-run. In the present study, we also consider the third question but in a multi-dimensional dynamic process.

The salient feature of the Cournot tatonnement process is that firms adjust output in response to the difference between their expectations and output observed in a market, and actual production takes place only when the expectation is identical with the observed output. A chaotic tatonnement, in spite of the use of output adjustment, does not converge to an equilibrium but fluctuates around it while remaining bounded. The chaotic tatonnement thus has a "problem" that no actual production is carried out as no convergence is brought about. There are, at least, two ways to deal with this problem : one is to stabilize or control chaos; the other is to allow intermediate production in the middle of the output adjustment process. In fact, research in the first direction has already begun; Bala, Majumder and Mitra [1] construct an exchange economy with a price adjustment based on the logistic map, and demonstrate the possibility of eliminating erratic price dynamics. Mendes and Mendes [10] introduce the pole-placement method into an overlapping generation model and demonstrate that aperiodic and very complicated economic motions in a twodimensional model can be turned into a stable steady state or into a regular cycle.

In this study, we take the latter approach (intermediate production during output adjustment) and give an answer to the third question. In particular, we construct a Cournot duopoly model and consider the economic implication of output chaos in a non-tatonnement process. A firm may be better off or worse off than at an equilibrium, depending on the prevailing market output. So our primary concern is to investigate whether, from the longrun point of view, the gain from the chaotic output instability can dominate the loss. Alternatively put, we are interested in answering the following question; Can chaos be more profitable than an equilibrium in a Cournot duopoly market?

This paper is organized as follows. Section 2 constructs a dynamic model of Cournot duopoly. Section 3 examines the existence of multiple equilibria and their stability. Section 4 , the main section, compares the long-run average profit along a chaotic trajectory with equilibrium profit analytically as well as numerically. Section 5 gives concluding remarks. All proofs are given in the Appendix.

\section{Nonlinear Duopoly Model}

In this section, we construct a discrete-time Cournot model in which an inverse demand function is assumed to be linear and decreasing,

$$
p=a-b Q, \quad a>0 \text { and } b>0,
$$

where $Q$ is the industry output, provided demand equals supply. To simplify the dynamic process, only two firms are considered in this study. These duopolists, denoted by $X$ and $Y$, produce homogenous outputs $x$ and $y$ so that the total supply is made up of supplies of the two duopolists, $x+y=Q$. Each firm is assumed to have a possibility of production externality in the sense that the choice by any one firm affects the production strategy of the other firm. Although externalities come in many varieties, we confine our analysis to the case in which the externality affects the cost of production. ${ }^{1}$ In particular, we deal with the 
simplest presence of externality in which the production cost of a firm is constant with respect to its own production and nonlinear with respect to its rival's production. Thus the cost functions of firms $X$ and $Y$ are specified by

$$
C^{x}(x, y)=c(y) x \quad \text { and } \quad C^{y}(y, x)=c(x) y .
$$

This specification implies that the marginal cost of production of one firm depends on the amount of output produced by the other firm. We say it has a positive externality if the marginal cost is decreasing for an increment of the rival's output and a negative externality if it is increasing.

Solving the profit maximization problems yields reaction functions of duopolists ; for firm $X$

$$
r^{x}\left(y^{e}\right)=\arg \max _{x} \Pi^{x}\left(x, y^{e}\right)
$$

and for firm $Y$,

$$
r^{y}\left(x^{e}\right)=\arg \max _{y} \Pi^{y}\left(x^{e}, y\right),
$$

where $x^{e}$ and $y^{e}$ are output expectations and the profit functions are defined as follows,

$$
\Pi^{x}\left(x, y^{e}\right)=\left(a-b\left(x+y^{e}\right)\right) x-c\left(y^{e}\right) x,
$$

and

$$
\Pi^{y}\left(y, x^{e}\right)=\left(a-b\left(x^{e}+y\right)\right) x-c\left(x^{e}\right) y .
$$

Due to the presence of production externality, the reaction function can be up- or downward-sloping according to whether the external effect is positive or negative. ${ }^{2}$ WL call a firm with upward-sloping reaction function an imitator who imitates the rival's behavior ; if the rival expands output, so does the imitator. They call a firm with downward-sloping reaction function an accommodator who accommodates the rival's behavior; the accommodator is a text-book duopolist and adapts to reduce residual demand if the rival expands output. Further, they call a firm with a unimodal reaction curve a dualist ; the dualist has a critical value of the rival's output and changes its strategic profile from the imitator to the accommodator if the rival expands output beyond the critical value. Although WL do not consider any economic situation in which a firm becomes a dualist, Kopel [7] takes the bookbuying-habit hypothesis ${ }^{3}$ and provides a possible microeconomic underpinning for which the profit maximization leads to an inverted $U$-shaped reaction function. However, as pointed out by WL, the inverted $U$-shaped reaction function passing through the origin has a

${ }^{1}$ We follow Kopel [7] who assumes a cost function incorporating the production externality.

${ }^{2}$ From the first-order condition of profit maximization, the slope of firm $X$ 's reaction function is given by

$$
\frac{d r^{x}\left(y^{e}\right)}{d y^{e}}=-\frac{1}{2}\left(b+c^{\prime}\left(y^{e}\right)\right),
$$

which is negative if $c^{\prime}>0$ (i.e., negative externality) and can be positive only if $c^{\prime}<0$ (i.e., positive externality). The same is true for firm $Y$.

${ }^{3}$ Suppose you start producing books. You can sell only a few books if only a few book stores exist, and people do not have a book-buying habit or if you are invisible among a huge number of book sellers. You can sell well if a moderate number of book stores exist. See Kopel [7] for more details. 
difficulty in rationalizing duopolist's behavior. It means that one firm offers no output in response to zero output of the rival, so that the monopoly's output is taken to be zero. In this study, we move one step forward and give microeconomic foundations for the occurrences of $U$-shaped as well as general inverted $U$-shaped reaction function. ${ }^{4}$

To examine effects of a production externality on the duopoly dynamics characterization, we specify the form of the marginal cost as

$$
c_{P}(z)=a-b z-2 b\left(1-(\sqrt{\alpha}(z-1)+1)^{2}\right),
$$

and

$$
c_{N}(z)=a-b z-2 b(\sqrt{\alpha}(z-1)+1)^{2},
$$

where $z$ denotes the rival's output. Here we assume $\frac{1}{64} \leq \alpha \leq 4$ and $a \geq 3 b$ for which $c_{P}(z)$ as well as $c_{N}(z)$ is non-negative for $0 \leq z \leq 1 .^{5}$ The curvatures of both functions is stronger for larger $\alpha$. Thus $\alpha$ can be thought as a proxy for the strength of the external effect.

$c_{P}(z)$ takes on a $U$-shaped profile and accounts for asymmetric production externality that turns to be positive to negative as the rival keeps expanding output. The book-buying habit hypothesis can justify the occurrence of this positive-negative production externality. On the other hand, $c_{N}(z)$ takes on an inverted $U$-shaped (i.e., mound-shaped) profile and implies that the marginal cost of production increases and then decreases as the rival's output $z$ increases. This shape accounts for another type of asymmetric externality, the negativepositive production externality, which may occur, for example, in the following interregional market. Two regions compete for exporting goods. Under strategic trade policy, the regional government grants export subsidy to a representing firm only if the rival's output exceeds a critical value. The per unit subsidy increases when the rival's output increases further. The "net" marginal cost of production of each firm is constant to its own but increasing to the rival's output because the firm employs labor from an interregional labor market and the wage rate increases if the amount of the demand (i.e., other firms' employment) increases. Assuming that the export subsidies eventually exceed the wage rate increment, the "total" marginal cost of the firm varies unimodal with respect to the rival's output.

Substituting (7) or (8) into (2) yields the following non-linear reaction functions,

$$
r_{P}(z)=1-(\sqrt{\alpha}(z-1)+1)^{2},
$$

and

$$
r_{N}(z)=(\sqrt{\alpha}(z-1)+1)^{2} .
$$

If $\alpha=1$ (i.e., the externality is rather week), $r_{P}$ is monotonically decreasing for $z$ and thus describes the accommodator's strategy. As $\alpha$ becomes larger, the shape of $r_{P}$ becomes

${ }^{4}$ Here "general" means that the reaction function does not necessarily pass through the origin and has a positive intercept.

${ }^{5}$ The minimum of $c_{P}(x)$ is non-negative if $\frac{a}{b} \geq 3+\frac{1}{8 \alpha}-\frac{1}{\sqrt{\alpha}} . \quad c_{N}(1) \geq 0$ if $a \geq 3 b$ and $c_{N}(0) \geq 0$ if $a \geq$ $3 b(\sqrt{\alpha}-1)^{2}$. If $\frac{1}{64} \leq \alpha \leq 4$ and $a \geq 3 b$, it can be verified that the all conditions above are satisfied. 
inverted $U$-shaped indicating that the firm turns into a dualist from an accommodator. Depending on the strength of the externality, the same firm can be a dualist or an accommodator so that we call such a firm an $A$-dualist. On the contrary, $r_{N}$ is monotonically increasing with $z$ for $\alpha=1$ and takes on $U$-shaped profile with increasing of $\alpha$. This implies that the firm can change into a dualist from an imitator if it has $c_{N}(z)$. For this reason, by analogy we call such a firm an $I$-dualist.

If firms have naive expectations, $x_{t}^{e}=x_{t-1}$ and $y_{t}^{e}=y_{t-1}$, the process of output adjustment is

$$
\left\{\begin{array}{l}
x_{t+1}=r^{x}\left(y_{t}\right) \\
y_{t+1}=r^{y}\left(x_{t}\right)
\end{array}\right.
$$

where the subscripts $P$ and $N$ are suppressed only for notational simplicity.

Intersections of these two reaction functions are Cournot-Nash equilibria at which each firm's expectation about the other firm's behavior is confirmed by the rival's actual behavior. If the expectation is not correct, then firms adjust their outputs.

Each of two firms can have either of two cost functions, $c_{P}$ in case of the positivenegative externality or $c_{N}$ in case of the negative-positive externality. Consequently, three distinct cases can be identified depending on which asymmetric externality is present. Case I : Both firms have the positive-negative externality and thus both are $A$-dualists. Case II : Both firms have the negative-positive externality and thus both are $I$-dualists. Case III : One firm has the positive-negative externality and the other firm has the negative-positive externality. One is $I$-dualist and the other is $A$-dualist. We confine attention to the homogenous instances of Case I and Case II in this study, and examine a heterogeneous example of Case III in Matsumoto and Nonaka [9]. We investigate the following problem : What can be said about the duopoly dynamics for the asymmetric pattern of production externality?

\section{Equilibrium}

In this section, we solve the dynamic equation for equilibrium points in Case I and Case II and clarify the stability properties of these equilibria in terms of the parameter $\alpha$.

We first determine the equilibrium points in Case I. Substituting (9) into (11) yields the following adjustment process,

$$
\left\{\begin{array}{l}
x_{t+1}=1-\left(\sqrt{\alpha}\left(y_{t}-1\right)+1\right)^{2}, \\
y_{t+1}=1-\left(\sqrt{\alpha}\left(x_{t}-1\right)+1\right)^{2} .
\end{array}\right.
$$

Cournot-Nash equilibrium is a point at which two reaction curves cross. Since reaction curves are nonlinear, multiple intersections are possible. Solving (12) for $x$ and $y$, we have four equilibria, as shown in Figure $1(\mathrm{a}),{ }^{6}$ which are denoted by $S_{1}^{I}=\left(s_{1}^{I}, s_{1}^{I}\right), S_{2}^{I}=\left(s_{2}^{I}, s_{2}^{I}\right), S_{3}^{I}=$ $\left(s_{3}^{I}, s_{4}^{I}\right)$ and $S_{4}^{I}=\left(s_{4}^{I}, s_{3}^{I}\right)$ where

\footnotetext{
${ }^{6}$ The superscript " $I$ " over $s_{i}$ and $S_{i}(i=1,2,3,4)$ is omitted in Figure 1 only for the notational simplicity.
} 


$$
\left\{\begin{array}{l}
s_{1}^{I}=\frac{2 \alpha-2 \sqrt{\alpha}-1-\sqrt{4 \sqrt{\alpha}+1}}{2 \alpha} \\
s_{2}^{I}=\frac{2 \alpha-2 \sqrt{\alpha}-1+\sqrt{4 \sqrt{\alpha}+1}}{2 \alpha} \\
s_{3}^{I}=\frac{2 \alpha-2 \sqrt{\alpha}+1+\sqrt{4 \sqrt{\alpha}-3}}{2 \alpha} \\
s_{4}^{I}=\frac{2 \alpha-2 \sqrt{\alpha}+1-\sqrt{4 \sqrt{\alpha}-3}}{2 \alpha}
\end{array}\right.
$$

It can be checked that $S_{1}^{I} \gtreqless 0$ corresponds to $\alpha \gtreqless 4$ and that $S_{3}^{I}$ and $S_{4}^{I}$ are symmetric and exist for $\alpha \geq \alpha_{1}=\frac{9}{16}$.

To determine the stability properties of an equilibrium point, we linearize the adjustment process (11) and get a Jacobian matrix,

$$
J=\left(\begin{array}{cc}
0 & \frac{d r^{x}}{d y} \\
\frac{d r^{y}}{d x} & 0
\end{array}\right)
$$

with the eigenvalues $\lambda_{1}=-\lambda_{2}=\lambda$ where $\lambda=\frac{d r^{x}}{d y} \frac{d r^{y}}{d x}$ and the derivatives are evaluated at the equilibrium. Thus the equilibrium is stable if and only if

$$
|\lambda|=\left|\frac{d r^{x}}{d y} \frac{d r^{y}}{d x}\right|<1
$$

We then have the following stability results in Case I.

Theorem 1 If the positive-negative externality is present, then (i) $S_{1}^{I}$ is unstable for all $\alpha>$ 0 ; (ii) $S_{2}^{I}$ is stable for $\alpha \leq \alpha_{1}=\frac{9}{16}$; (iii) $S_{3}^{I}$ and $S_{4}^{I}$ are stable for $\alpha_{1} \leq \alpha \leq \alpha_{2}=\frac{25}{16}$.

Proof. See the Appendix.

These results are illustrated in Figure 1(b) where the bold lines indicate stable equilibria and the dotted lines unstable equilibria. For $\frac{25}{16}<\alpha \leq 4$, all equilibria are unstable, and a trajectory does not converges to any of the four equilibria but keeps fluctuating within the limited region, $[0,1] \times[0,1]$. Since $s_{1}^{I}>0$ for $\alpha>4$, there is a shaded region of $(x, y)$ denoted by $E_{1}$ in Figure 1 (a) where we set $\alpha=5$. Any trajectory starting from a point that does not belong to $E_{1}$ is trapped into this region after finite iterations and then diverges to negative infinity, that is, the duopoly economy self-destructs. Since we are not interested in such a self-destructive economy, we confine our attention to the case of $\alpha \leq 4$ below.

We now consider Case II where the negative-positive externality is present and firms are $I$-dualists. Substituting (10) into (11) yields the following adjustment process,

$$
\left\{\begin{array}{l}
x_{t+1}=\left(\sqrt{\alpha}\left(y_{t}-1\right)+1\right)^{2}, \\
y_{t+1}=\left(\sqrt{\alpha}\left(x_{t}-1\right)+1\right)^{2} .
\end{array}\right.
$$




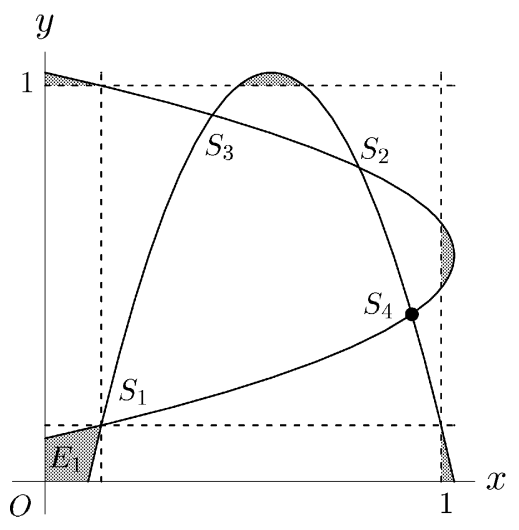

(a) Reaction functions

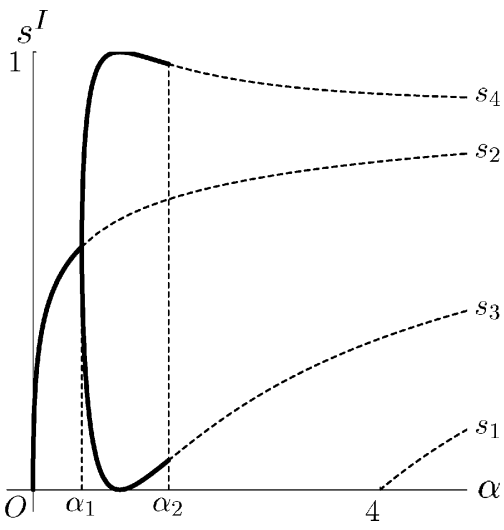

(b) Variation of equilibrium outputs

Figure 1. Multiple equilibria in Case I

where each reaction function has a $U$-shaped curve with respect to the rival's output. Solving this for $x$ and $y$ (i.e., $x=x_{t}=x_{t+1}$ and $y=y_{t}=y_{t+1}$.) gives the following four equilibria, as shown in Figure $2(\mathrm{a}),{ }^{7} S_{1}^{I I}=\left(s_{1}^{I I}, s_{2}^{I I}\right), S_{2}^{I I}=\left(s_{2}^{I I}, s_{2}^{I I}\right), S_{3}^{I I}=\left(s_{3}^{I I}, s_{4}^{I I}\right)$ and $S_{4}^{I I}=\left(s_{4}^{I I}, s_{3}^{I I}\right)$ where

$$
\left\{\begin{array}{l}
s_{1}^{I I}=1, \\
s_{2}^{I I}=\frac{(1-\sqrt{\alpha})^{2}}{\alpha}, \\
s_{3}^{I I}=\frac{2 \alpha-2 \sqrt{\alpha}-1+\sqrt{(2 \sqrt{\alpha}+1)(2 \sqrt{\alpha}-3)}}{2 \alpha} \\
s_{4}^{I I}=\frac{2 \alpha-2 \sqrt{\alpha}-1-\sqrt{(2 \sqrt{\alpha}+1)(2 \sqrt{\alpha}-3)}}{2 \alpha} .
\end{array}\right.
$$

It can be checked that $S_{2}^{I I}$ exists for any $\alpha \geq 0$ and that $S_{3}^{I I}$ and $S_{4}^{I I}$ exist for $\alpha \geq \frac{9}{4}$. Applying the condition given in (15) to the linearized matrix of (16), we have the following stability results in Case II,

Theorem 2 If the negative-positive externality is present, then (i) $S_{1}^{I I}$ is stable for $\alpha \leq \alpha_{0}^{\prime}=\frac{1}{4}$;

(ii) $S_{2}^{I I}$ is stable for $\alpha_{0}^{\prime} \leq \alpha \leq \alpha_{1}^{\prime}=\frac{9}{4}$; (iii) $S_{3}^{I I}$ and $S_{4}^{I I}$ are stable for $\alpha_{1}^{\prime} \leq \alpha \leq \alpha_{2}^{\prime}=\frac{7}{4}+\sqrt{\frac{3}{2}} \simeq 2.97$.

Proof. See the Appendix.

These results are illustrated in Figure 2 (b) where the meaning of the lines are the same

\footnotetext{
7 The superscript " $I I$ " over $s_{i}$ and $S_{i}(i=1,2,3,4)$ is omitted in Figure 2 only for the notational simplicity.
} 


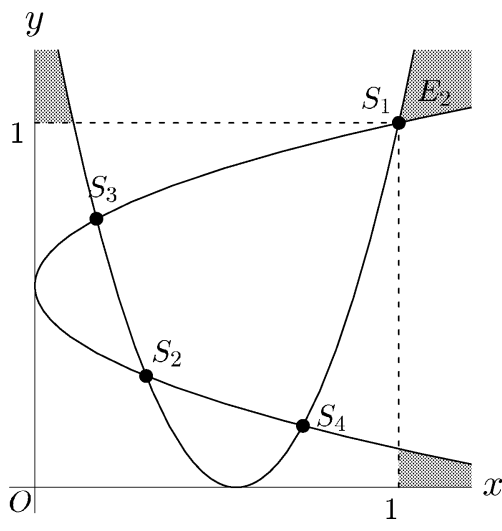

(a) Reaction functions

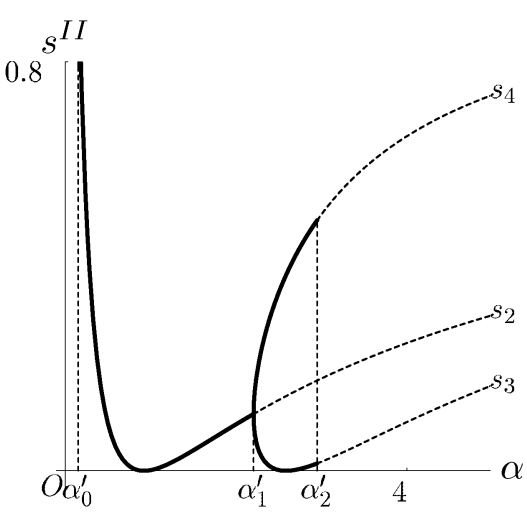

(b) Variation of equilibrium outputs

Figure 2. Multiple equilibria in Case II

as in Figure 1(b). For $\frac{7}{4}+\sqrt{\frac{3}{2}}<\alpha \leq 4$, all stationary states are unstable and trajectories chaotically fluctuate. Since $r_{N}(0)>1$ for $\alpha>4$, there is a shaded region of $(x, y)$ denoted by $E_{2}$ in Figure 2 (a) where we set $\alpha=5$, and any trajectory starting from a point in $[0,1] \times[0,1]$ is trapped into this region after finite iterations and then diverges to positive infinity, that is, the economy again self-destructs. With the assumption $\alpha \leq 4$, we can omit further considerations on the self-destructive economy in Case II.

\section{Long-run Average Behavior}

In this section, we present the main results concerning implications of chaotic dynamics generated in the nonlinear duopoly model. In particular, we present two examples to consider the meaning of the long-run behavior of chaotic duopolists. Setting $\alpha=4$ in the first example and $\alpha<4$ in the second example, we clarify an economic meaning of chaos analytically and numerically with the following three steps. First, we calculate each firm's profit earned at an equilibrium point, which we call the equilibrium profit. Second, we calculate the long-run average profit of each firm taken along the chaotic trajectory. Finally, we compare the long-run average profit with the equilibrium profit to check which is greater.

In most of this section, dynamics characterizations are considered in the situation where firms are homogenous with respect to their reaction function with the same value of parameter. In the latter half of Section 4.2, we focus attention to heterogeneous situation in which firms have the same reaction function but different values of parameter $a$. In such a case, we find that the chaotic long-run average profit can be greater than the equilibrium profit.

\subsection{Analytical Results for $\alpha=4$}

Throughout this subsection $\alpha=4$ is used. We divide this section into two and consider Case I in the first half and Case II in the second half. 


\subsubsection{Case I : Positive-Negative Externality}

Substituting (13) into $\Pi_{x}(x, y)$ and $\Pi_{y}(x, y)$ gives the equilibrium profits in Case I.

$$
\left\{\begin{array}{l}
\prod_{x_{1}}^{*}=\prod_{y_{1}}^{*}=0 \text { at } S_{1}^{I}, \\
\prod_{x_{2}}^{*}=\prod_{y^{2}}^{*}=\frac{9 b}{16} \text { at } S_{2}^{I}, \\
\prod_{x_{3}}^{*}=\frac{5(3-\sqrt{5}) b}{32}<\prod_{y_{3}}^{*}=\frac{5(3+\sqrt{5}) b}{32} \text { at } S_{3}^{I}, \\
\prod_{x_{4}}^{*}=\frac{5(3+\sqrt{5}) b}{32}>\prod_{y_{4}}^{*}=\frac{5(3-\sqrt{5}) b}{32} \text { at } S_{4}^{I} .
\end{array}\right.
$$

If we rank four equilibria according to profits and use the symbol $>$ to mean that one state is strictly preferred to another, the preference ordering of firm $X$ is

$$
S_{4}^{I}>S_{2}^{I}>S_{3}^{I}>S_{1}^{I}
$$

and, as $S_{3}^{I}$ and $S_{4}^{I}$ are symmetric, the preference ordering of firm $Y$ is obtained by replacing $S_{4}^{I}$ with $S_{3}^{I}$,

$$
S_{3}^{I}>S_{2}^{I}>S_{4}^{I}>S_{1}^{I}
$$

Substituting $\alpha=4$ into (11) and arranging terms yield the reaction functions,

$$
\left\{\begin{array}{l}
r\left(y_{t}\right)=4 y_{t}\left(1-y_{t}\right), \\
r\left(x_{t}\right)=4 x_{t}\left(1-x_{t}\right),
\end{array}\right.
$$

which are logistic maps. Substituting further these reaction functions into the adjustment process (11), gives a dynamic system of double logistic map. It has been demonstrated that the double logistic map can generate chaos. Let $\left\{\left(x_{t}, y_{t}\right)\right\}_{t=0}^{\infty}$ be a chaotic trajectory of output and let $\left\{\Pi\left(x_{t}, y_{t}\right)\right\}_{t=0}^{\infty}$ be the corresponding trajectory of profit associated with this output trajectory. Although the adjustment process is two-dimensional, dynamical properties are characterized by a one-dimensional map. The whole trajectory can be decomposed into two sub-trajectories, $\left\{\left(x_{2 t}, y_{2 t}\right)\right\}_{t=0}^{\infty}$, the even-period trajectory, and $\left\{\left(x_{2 t+1}, y_{2 t+1}\right)\right\}_{t=0}^{\infty}$, the oddperiod trajectory. If the initial point $\left(x_{0}, y_{0}\right)$ is chosen to satisfy $y_{0}=r\left(x_{0}\right)$, then it can be verified that every even-period point $\left(x_{2 t}, y_{2 t}\right)$ is located on the locus of $y=4 x(1-x)$ and every odd-period point $\left(x_{2 t+1}, y_{2 t+1}\right)$ is located on the locus of $x=4 y(1-y)$. That is, the dynamics of the sub-trajectories can be described by the one-dimensional logistic map, $r(x)$ or $r(y)$. The same procedure can be applied to the profit trajectory.

The average is a basic statistical property of the chaotic dynamics. Although it is generally impossible to calculate an average of infinite elements, we can obtain the average profit of the chaotic trajectory, $\left\{\Pi\left(x_{t}, y_{t}\right)\right\}_{t=0}^{\infty}$, in the case of $\alpha=4$ with the help of the Mean Ergodic theorem of Brikhoff-von Neuman, taking account of the following properties of the logistic map $^{8}$;

Theorem 3 For $r(x)=4 x(1-x):[0,1] \rightarrow[0,1]$ we have the following results : (i) It generates ergodic chaos. (ii) There is only one absolutely continuous invariant ergodic measure on unit interval. (iii) The explicit form of the density function is given by

\footnotetext{
8 These are well known properties; see, for example, Day [5] for details.
} 


$$
\Phi(x)=\frac{1}{\pi \sqrt{x(1-x)}} .
$$

Consider, for examples, the even-period trajectory, $\left\{\Pi\left(x_{2 t}, y_{2 t}\right)\right\}_{t=0}^{\infty}$, where each point $\left(x_{2 t}\right.$, $\left.y_{2 t}\right)$ satisfies $y_{2 t}=r\left(x_{2 t}\right)$ and its density is the same as $\Phi(x)$. Since the reaction function with $\alpha=4$ is ergodic, the time average of the even-period profits converges to its space average, namely, the mean value of profit evaluated on the domain of output,

$$
\lim _{T \rightarrow \infty} \frac{1}{T} \sum_{t=0}^{T-1} \Pi\left(x_{2 t}, r\left(x_{2 t}\right)\right)=\int_{0}^{1} \tilde{\Pi}(x) \Phi(x) d x,
$$

where $\Pi(x, r(x))=\tilde{\Pi}(x)$. Similarly, it is possible to calculate the average profit of the oddperiod profit. An average of these two average profits is the long-run average profit obtained along chaotic trajectory $\left\{\Pi\left(x_{t}, y_{t}\right)\right\}_{t=0}^{\infty}$. This is summarized in the following theorem.

Theorem 4 Let $\left\{\left(x_{t}, y_{t}\right)\right\}_{t=0}^{\infty}$ be the trajectory of output generated by the adjustment process of (21). Then the long-run average profit is

$$
\bar{\Pi}:=\lim _{T \rightarrow \infty} \frac{1}{T} \sum_{t=0}^{T-1} \Pi\left(x_{t}, y_{t}\right)=\frac{b}{4} .
$$

Proof. See the Appendix.

So far, we have obtained the equilibrium profit at each equilibrium and the long-run average profit taken along a chaotic trajectory whose ranking is

$$
\frac{5(3+\sqrt{5}) b}{32}>\frac{9 b}{16}>\frac{b}{4}>\frac{5(3-\sqrt{5}) b}{32} \text {. }
$$

From (19), (20) and (23), for firm $X, S_{3}^{I}$ and $S_{2}^{I}$ are better than chaotic fluctuations but $S_{4}^{I}$ and $S_{1}^{I}$ are worse while for firm $Y, S_{4}^{I}$ and $S_{2}^{I}$ are better than chaotic fluctuations but $S_{3}^{I}$ and $S_{1}^{I}$ are worse. Since all Cournot-Nash equilibria are unstable for $\alpha=4$, the market force (i.e., the output adjustment) takes neither of two firms to their preferable state. As stated in Introduction, controlling chaos (i.e., the fine tuning of the dynamic system) can, if it is successfully operated, eliminate those output fluctuations and turns the equilibrium point from the unstable one into a stable one. However, it creates another problem, that is, the selection of equilibrium points. For firm $X$, the best choice is $S_{4}^{I}$ which is the worst choice for firm $Y$. On the other hand, $S_{3}^{I}$ is the most preferable equilibrium for firm $Y$ and the least preferable for firm $X . \quad S_{2}^{I}$ is better than chaotic fluctuations but is the second-best for both firms. No matter which equilibrium is attained through a control of chaos, there always exist an incentive to deviate from it. In other words, there is no way to "aggregate" individual ordering to make one social ordering in the chaotic duopoly market.

4.1.2 Case II : Negative-Positive Externality

Using an approach similar to that in Section 4.1.1 and substituting (17) into each of $\Pi_{x}(x, y)$ and $\Pi_{y}(x, y)$ gives the equilibrium profits in Case II, 


$$
\left\{\begin{array}{l}
\prod_{x 1}^{*}=\prod_{y 1}^{*}=b \text { at } S_{1}^{I I}, \\
\prod_{x 2}^{*}=\prod_{y 2}^{*}=\frac{b}{16} \text { at } S_{2}^{I I}, \\
\prod_{x 3}^{*}=\frac{(7-3 \sqrt{5})^{2} b}{64}<\prod_{y 3}^{*}=\frac{(7+3 \sqrt{5})^{2} b}{64} \text { at } S_{3}^{I I}, \\
\prod_{x^{*}}^{*}=\frac{(7+3 \sqrt{5})^{2} b}{64}>\prod_{y 4}^{*}=\frac{(7-3 \sqrt{5})^{2} b}{64} \text { at } S_{4}^{I I} .
\end{array}\right.
$$

We have the following ordering among four equilibria and the chaotic fluctuations,

$$
b>\frac{(7+3 \sqrt{5}) b}{32}>\frac{b}{4}>\frac{b}{16}>\frac{(7-3 \sqrt{5}) b}{32} .
$$

Contrary to Case I, we have the dominant strategy $S_{1}^{I I}$ in Case II, which is the best choice for both firms but is the extreme equilibrium as shown in Figure $2(\mathrm{a})$. If we eliminate $S_{1}^{I I}$ from further considerations, the same problem arises among the inner equilibria ; the best choice of one firm is the worst choice for the other. Putting this differently, chaotic fluctuation is profitable for one firm but is disadvantageous for the other.

\subsection{Simulation Results for $\alpha<4$}

We have shown that chaotic fluctuations can be profitable for one of two duopolists when $\alpha=4$. The question naturally arises as to what can be said about the long-run average profit when $\alpha<4$ ? One difficulty to deal in the case of $\alpha<4$ is that explicit forms of densities for chaotic trajectories are unable to be constructed. Consequently, the Mean Ergodic Theorem is not applicable to derive the long-run average profits. Therefore, in this sub-section, we take a numerical approach: we perform numerical simulations in order to investigate whether the theoretical results still hold for $\alpha<4$. In particular, we directly calculate the time average of profit over the first $T$ periods along a chaotic trajectory, and then compare these with the equilibrium profits. We investigate the homogenous case where firms have the same reaction functions and the same parameter values and also the heterogenous case where firms have the same reaction functions but different parameter values.

\subsubsection{Homogenous Firms}

Before proceeding, we need to check whether a finite average (i.e., numerical average) can approximate an infinite average (i.e., theoretical average). Figure 3 illustrates how finite averages of firm $X$ "converge" to the long-run averages when $\alpha=4$. In order to better indicate the quality of the convergence process, we have drawn a dotted horizontal line at $\bar{\Pi}_{X_{I}}$, the equilibrium profit of firm $X$ in Case $I$ in Figure $3(\mathrm{a})$ and the one at $\bar{\Pi}_{X_{I I}}$, the equilibrium profit in Case II in Figure 3 (b). There, it can be seen that the numerical average approaches the theoretical value as the number of periods used to calculate the finite average increases. It is, therefore, reasonably to assume that the finite average almost equals the theoretical average if the number of iterations exceeds 1,000. Now we are ready to conduct numerical experiments to check whether the theoretical results can hold when $\alpha<4$. We focus on the average behavior of firm $X$ below. Since firms are homogenous, the same results can be obtained for firm $Y$. Figure 4 (a), respectively Figure 4 (b), gives numerical results in Case I where firm $X$ has the inverted $U$-shaped reaction function, respectively 


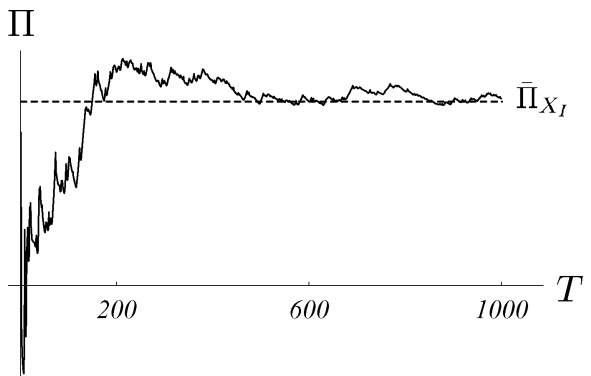

(a) Case I

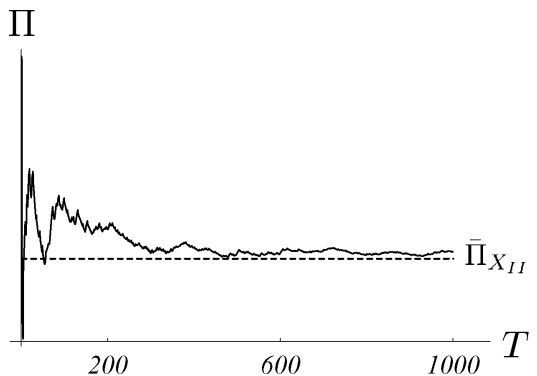

(b) Case II

Figure 3. Convergence of the numerical average

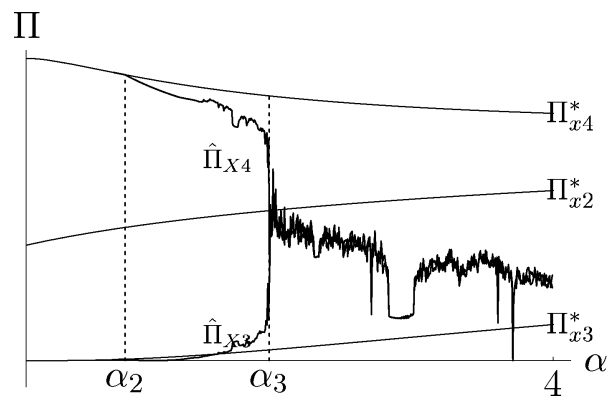

(a) Case I

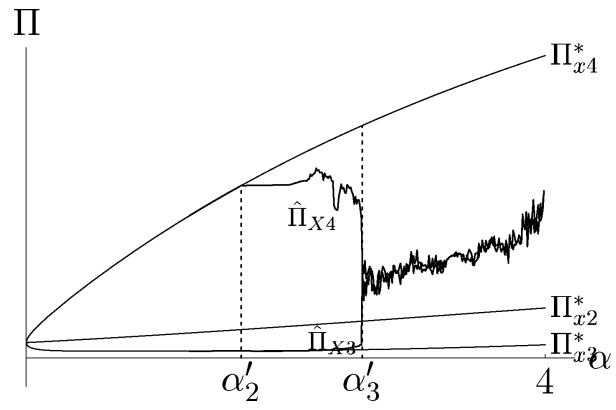

(b) Case II

Figure 4. Variation of the average profit with respect to $1<\alpha<4$

Case II where firm $X$ has the $U$-shaped reaction function. Since both figures give qualitatively the same results so we focus on Figure 4 (a). Two different trajectories of the average profit, $\hat{\Pi}_{x_{3}}$ and $\hat{\Pi}_{x_{4}}$, are depicted.

(i) As shown in Theorem 1 (iii), $S_{3}$ and $S_{4}$ are stable for $\alpha \leq \alpha_{2}$, the average profit converges to the equilibrium profits. Consequently, the average profit lines are invisible (or identical with the equilibrium profit lines).

(ii) $\quad \alpha_{2}$ is a bifurcation point at which a stable equilibrium become unstable. For $\alpha_{2}<$ $\alpha \leq \alpha_{3}$, two average profit lines starts to deviate from the equilibrium lines. If we rank the profits, $\prod_{x_{4}}^{*}>\prod_{x_{4}}>\prod_{x_{2}}^{*}>\prod_{x_{3}}^{*}$, although a trajectory fluctuates for $\alpha>\alpha_{2}$, there are disjoint two attractors, one is located in a neighborhood of $S_{3}$ and the other in a neighborhood of $S_{4}$. The diagram indicates that the average profit obtained around $S_{4}$ is larger than the one around $S_{3}$, i.e. $\hat{\Pi}_{x 4}>\hat{\Pi}_{x 3}$.

(iii) For $\alpha=\alpha_{3}$, two attractors start to merge into one. For $\alpha>\alpha_{3}$ a trajectory starting near one equilibrium frequently visits a neighborhood of the other equilibrium so the two average profits are getting closer. Regardless of the choice of the initial points, the average profit converges to the same value. As $\alpha$ increases further to 4 , the two averages converge to their theoretical value.

From these observations, we can say that the average profit is higher for one firm and 


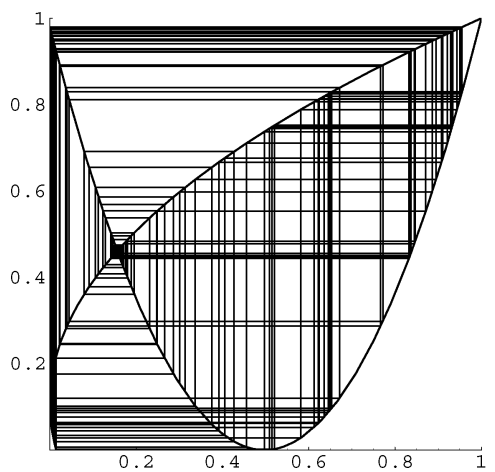

(a) Reaction functions

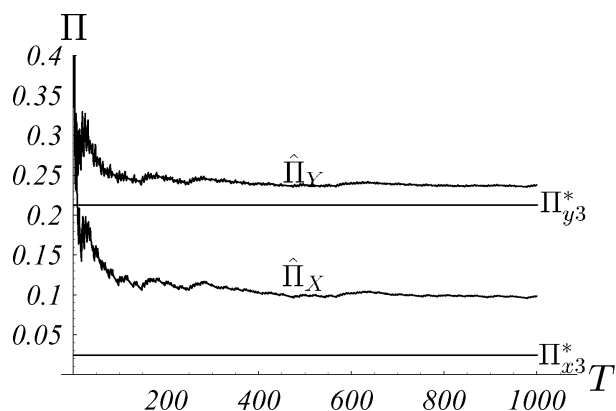

(b) Convergence of the numerical average

Figure 5. Chaos is profitable than the Nash-equilibrium

lower for the other for $\alpha>\alpha_{2}$. This numerical result is the same as the previously obtained theoretical result.

\subsubsection{Heterogenous Firms}

Homogenous firms have been assumed so far. We relax this assumption somewhat and consider the effect of different or heterogeneous firms on the long-run behavior. Although there are many ways to introduce "heterogeneity" into the model, we take one special example in which both firms are $I$-dualists but parameter values of their reaction functions are different. Let $\alpha=1.26$ for firm $X$ and $\alpha=3.96$ for firm $Y$. In Figure 5(a), the $U$ shaped curve is the reaction function of firm $Y$, and the upward-sloping curve is the reaction function of firm $X$. These two curves intersect only twice, which means there are two Cournot-Nash equilibria under given values of parameters. Returning to (16), we find the two equilibria are $S_{1}$ and $S_{3}$. Since $S_{1}$ is an extreme point and unstable, trajectories fluctuate around $S_{3}$. In this asymmetric circumstance, we obtain new insights on the chaotic dynamics properties as shown in Figure 5. Figure 5 (a) illustrates the return map and suggests that the adjustment process is chaotic. Figure 5(b) depicts the average profits of two firms as a function of the number of iterations and the equilibrium profit (horizontal) lines. $\prod_{y 3}^{*}$ and $\Pi_{x 3}^{*}$ are the equilibrium profits of firm $X$ and firm $Y$ respectively. $\hat{\Pi}_{y}$ and $\hat{\Pi}_{x}$ denote the average profits. The average profit curves have sharp fluctuations in the beginning but gradually converge to some limit values as the number of iteration increases. It can be seen that for both the firms, the average profit curves are located above the corresponding equilibrium profit lines for any value of $T$, that is, the average profit is larger than the equilibrium profit. This numerical example indicates a strong possibility that both firms can benefit in chaotic market if some heterogeneities are present.

\section{Concluding Remarks}

This study presents a Cournot duopoly market in which the non-monotonic adjustment process of output may be chaotic. To construct an economic foundations of non-monotonic reaction functions, the investigation takes account of two patterns of asymmetric production 
externalities; the positive-negative externality and the negative-positive externality. To find an economic meaning of chaotic fluctuations, the study focuses on a case in which actual productions take place even if the expectations are not satisfied. This study calculates a long-run average profit taken along a chaotic trajectory and then compares it with an equilibrium profit taken at a Cournot-Nash equilibrium to check which can be larger from the long-run point of view.

The investigation in this study yields two main results and one interesting insight.

(i) If $\alpha=4$, the long-run average profit can be derived theoretically with the help of the Mean Ergodic Theorem. Comparing it with each of multiple equilibrium profits, we find that one firm benefits in a chaotic market but the other comes to harm. (ii) If $\alpha<4$, the long-run average profit cannot be derived as the Mean Ergodic Theorem is not applicable. However, qualitatively the same result as in (i) is confirmed by performing numerical simulations. In addition, by taking away the assumption of homogenous firms but introducing the one of heterogenous firms, we can demonstrate with numerical simulations that both duopolits can benefit in a chaotic market. Since chaotic trajectories do not converge to an equilibrium state, these are thought to be an unfavorable phenomenon in traditional economics. The results obtained in this study, in particular, the last numerical result, throw interesting light on the nature of complex dynamics.

Finally, we discuss very briefly some important respects in which our model needs to be extended. First, a real two-dimensional adjustment process should be considered. The process examined in this study is actually two independent process, which can be reduced to one-dimensional process. One possible alternative is a duopoly model with adaptive expectations that can not be decomposed into independent maps of the first order. A second extension concerns international trade in which Cournot competition often appears. Brander and Spencer [3] apply a quantity-competition model to an open economy where two countries compete in quantities in a third country and show that when the home government intends to shift monopolistic rent from foreign to domestic firms, an export subsidy is optimal. The vast amount of contributions on strategic trade policy appears since this pioneering paper. However, in the strategic international trade literature, the linear and stable assumptions have been conveniently adapted. As an importance of nonlinearity is recognized, introducing nonlinear reaction functions considered in the model that we have presented would increase the applicability of the nonlinear economic dynamic model considerably in the studies of international trade as well as interregional trade.

\section{Appendix : Proofs}

\section{A.1 Proof of Theorem 1}

Using (12), (15) can be rewritten as

$$
|\lambda|=\left|4 \alpha\left(\sqrt{\alpha}\left(y^{*}-1\right)+1\right)\left(\sqrt{\alpha}\left(x^{*}-1\right)+1\right)\right|<1
$$

where $\left(x^{*}, y^{*}\right)$ is a pair of equilibrium output.

(i) Setting $x^{*}=y^{*}=s_{1}^{I}$ gives

$$
\lambda=(1+\sqrt{1+4 \sqrt{\alpha}})^{2} .
$$


This is always greater than unity for arbitrary $\alpha$.

(ii) Setting $x^{*}=y^{*}=s_{2}^{I}$ and solving for $\alpha$, we have $\alpha<\frac{9}{16}$.

(iii) Setting $x^{*}=s_{3}^{I}$ and $y^{*}=s_{4}^{I}$ and applying the same procedure as with $S_{2}^{I}$, we have the stability condition of $S_{3}^{I}$ as $\frac{9}{16}<\alpha<\frac{25}{16}$. Since $S_{4}^{I}$ is symmetric to $S_{3}^{I}$, we have the same stability condition for $S_{4}^{I}$.

\section{A.2 Proof of Theorem 2}

Using (16), we also have (26) as the stability condition of Case II.

(i) Setting $x^{*}=y^{*}=s_{1}^{I I}$ gives $|\lambda|=|4 \alpha|$. We thus have $\alpha<\frac{1}{4}$.

(ii) Setting $x^{*}=y^{*}=s_{2}^{I I}$ and solving (26) for $\alpha$, we have the stability condition of $S_{2}^{I I}$ as $\frac{1}{4}<\alpha<\frac{9}{4}$.

(iii) Setting $x^{*}=s_{3}^{I I}$ and $y^{*}=s_{4}^{I I}$ and applying the same procedure, then we have the stability condition of $S_{3}^{I}$ as $\frac{9}{4}<\alpha<\frac{7}{4}+\sqrt{\frac{3}{2}}$ which is also the stability condition for $S_{4}^{I I}$.

\section{A.3 Proof of Theorem 3}

We calculate the long-run average profit for firm $X$. Since firms are homogeneous, the long-run average profit of firm $Y$ is the same as the one of firm $X$. Suppose that firm $X$ adjusts its output in odd periods and $Y$ adjusts the output in even periods. Then:

$$
x_{2 t+1}=x_{2(t+1)} \text { and } y_{2 t}=y_{2 t+1}, \quad n=0,1, \cdots
$$

Therefore from (11), we get:

$$
\begin{aligned}
& \left\{\left(x_{t}, y_{t}\right)\right\}_{t=0}^{\infty} \\
& \quad=\left\{\left(x_{0}, r^{y}\left(x_{0}\right)\right),\left(r^{x}\left(x_{0}\right), y_{1}\right), \cdots,\left(x_{2 t}, r^{y}\left(x_{2 t-1}\right)\right),\left(r^{x}\left(y_{2 t}\right), y_{2 t+1}\right), \cdots\right\} \\
& \quad=\left\{\left\{\left(r^{x}\left(y_{2 t+1}, y_{2 t+1}\right)\right\}_{t=0}^{\infty},\left\{\left(x_{2 t}, r^{y}\left(x_{2 t}\right)\right)\right\}_{t=0}^{\infty}\right\} .\right.
\end{aligned}
$$

Then :

$$
\begin{aligned}
\lim _{T \rightarrow \infty} & \frac{1}{T} \sum_{i=0}^{T-1} \Pi^{x}\left(x_{t}, y_{t}\right) \\
= & \frac{1}{2}\left(\lim _{n \rightarrow \infty} \frac{1}{n} \sum_{i=0}^{n-1} \Pi^{x}\left(r^{x}\left(y_{2 n+1}\right), y_{2 n+1}\right)+\lim _{n \rightarrow \infty} \frac{1}{n} \sum_{i=0}^{n-1} \Pi^{x}\left(x_{2 n}, r^{y}\left(x_{2 n}\right)\right)\right) \\
= & \frac{1}{2}\left(\int_{0}^{1} \Pi^{x}\left(r^{x}(u), u\right) \Phi(0) d u+\int_{0}^{1} \Pi^{x}\left(u, r^{y}(u)\right) \Phi(u) d u\right) \\
= & \frac{b}{4} .
\end{aligned}
$$

Those calculations derive same results in both of Case I and II.

\section{Acknowledgement}

Helpful comments by two annoymous referees are gratefully acknowledged. Financial supports from Chuo University (Joint Research Grant 0382) is highly appretiated. 


\section{References}

[1 ] Bala, V., Majumdar, M. and Mitra, T., “A Note on Controlling a Chaotic tatonnement,” Journal of Economic Behavior \& Organization, Vol. 33, 1998, pp. 411-420.

[2] Bischi, G.I. and Kopel, M., "Equilibrium Selection in a Nonlinear Duopoly Game with Adaptive Expectations,” Journal of Economic Behavior \& Organization, Vol. 46, 2001, pp. 73-100.

[ 3 ] Brander, J.A. and Spencer, B.J., "Export Subsidies and International Market Rivalry,” Journal of International Economics, Vol. 18, 1985, pp. 83-100.

[ 4 ] Dana, R. and Montrucchio, L., "Dynamic Complexity in Duopoly Games," Journal of Economic Theory, Vol. 40, 1986, pp. 161-185.

[ 5 ] Day, R., Complex Economic Dynamics, MIT Press, 1994.

[6] Huang, W., "Caution Implies Profit," Journal of Economic Behavior \& Organization, Vol. 27, No. 2, 1995, pp. 257-277.

[7] Kopel, M., "Simple and Complex Adjustment Dynamics in Cournot Duopoly Models," Chaos, Solitons \& Fractals, Vol. 7, No. 12, 1996, pp. 2031-2048.

[8] Matsumoto, A., "Let It Be: Chaotic Price Instability can be Beneficial," Chaos, Solitons \& Fractals, Vol. 18, 2003, pp. 745-758.

[9] Matsumoto, A. and Nonaka, Y., "Do Heterogeneous Duopolists Prefer Chaos?,” Proceedings of International Symposium on Nonlinear Theory and its Applications, 2002.

[10] Mendes, V. and Mendes, D.A., "Control of Chaotic Dynamics in an OLG Economic Model," to appear in Journal of Physics Conference Series, 2005.

[11] Puu, T., "Chaos in Duopoly Pricing," Chaos, Solitons \& Fractals 1, No. 6, 1991, pp. 573-531.

[12] Puu, T., “The Chaotic Duopolists Revisited," Journal of Economic Behavior \& Organization, Vol. 33, 1998, pp. 385-394.

[13] Rand, D., "Exotic Phenomena in Games and Duopoly Models," Journal of Mathematical Economics, Vol. 5, 1978, pp. 173-184.

[14] Witteloostuijn, A. and Lier, A., "Chaotic Patterns in Cournot Competition," Metroeconomica, Vol. 41, No. 2, 1991, pp. 161-185. 